\title{
Diacronie
}

Studi di Storia Contemporanea

$N^{\circ} 26,2 \mid 2016$

Un bilancio della scommessa democratica della Chiesa cattolica

\section{Francesca Fauri, Storia economica delle migrazioni italiane}

Jacopo Bassi

\section{(2) OpenEdition \\ Journals}

Edizione digitale

URL: http://journals.openedition.org/diacronie/4035

DOI: $10.4000 /$ diacronie.4035

ISSN: 2038-0925

Editore

Association culturelle Diacronie

Notizia bibliografica digitale

Jacopo Bassi, «Francesca Fauri, Storia economica delle migrazioni italiane », Diacronie [Online], № 26, 2 I

2016, documento 13, Messo online il 29 juin 2016, consultato il 24 septembre 2020. URL : http://

journals.openedition.org/diacronie/4035; DOI : https://doi.org/10.4000/diacronie.4035 


\section{RECENSIONE:}

\section{Francesca FAURI, Storia economica delle migrazioni italiane, Bologna, Il Mulino, 2015, 233 pp.}

a cura di Jacopo BASSI *

Esistono molte prospettive attraverso cui studiare l'emigrazione italiana: quella scelta da Francesca Fauri nel suo nuovo libro prende in esame l'aspetto economico dell'emigrazione italiana.

L'autrice insegna Storia economica nell'Università di Bologna e nei suoi precedenti lavori si è concentrata su temi di storia economica novecentesca. Dopo essersi occupata di integrazione economica europea ${ }^{1}$ si è orientata sul secondo dopoguerra analizzando nel dettaglio il Piano Marshall con particolare attenzione al caso italiano ${ }^{2}$. È dedicato all'economia italiana postbellica anche il manuale, scritto assieme a Patrizia Battilani, Mezzo secolo di economia italiana 1945-20083.

Con Storia economica delle migrazioni italiane, Fauri affronta prevalentemente il periodo precedente: l'ultima parte dell'Ottocento e la prima metà del Novecento. Il tema dell'emigrazione è sempre al centro dell'attenzione degli studiosi4, ma Francesca Fauri si è concentrata su un aspetto specifico: quello delle ragioni economiche della migrazione. Per avviare la sua analisi l'autrice parte da due considerazioni, dimostrate dai dati e da una solida letteratura di riferimento. La prima di queste è che la popolazione italiana non sia mai stata sedentaria, bensì abbia mostrato una

${ }^{1}$ FAURI, Francesca, L’integrazione economica europea, Bologna, Il Mulino, 2006.

2 ID., Il Piano Marshall e l'Italia, Bologna, Il Mulino, 2010.

3 ID., Mezzo secolo di economia italiana: 1945-2008, Bologna, Il Mulino, 2014.

4 Tra gli altri, recentemente editi, si vedano i lavori - incentrati sul ruolo delle migrazioni italiane in un'ottica di lungo periodo - di Paola Corti: CORTI, Paola, Storia delle migrazioni internazionali, Roma-Bari, Laterza, 2007; CORTI, Paola, SANFILIPPO, Matteo, L'Italia e le migrazioni, Roma-Bari, Laterza, 2012. Una prospettiva di lungo periodo si ritrova anche in: AUDENINO, Patrizia, TIRABASSI, Maddalena, Migrazioni italiane. Storia e storie dall'Ancien régime ad oggi, Milano, Bruno Mondadori, 2008. Intorno ad un periodo specifico e ad un tema si è, invece, soffermato Sandro Rinauro: RINAURO, Sandro, Il cammino della speranza. L'emigrazione clandestina degli italiani nel secondo dopoguerra, Torino, Einaudi, 2014. 
propensione allo spostamento per ragioni economiche; il secondo è che alcune figure nomadi, lavoratori itineranti, migranti e girovaghi - abbiano giocato il ruolo di apripista nelle grandi migrazioni internazionali. Il riferimento è a quei lavoratori come il Rodmund descritto da Levis, stagnino, o i carbonai delle prealpi lombarde di cui racconta Cassola ${ }^{6}$.

Il libro, a partire da queste premesse, si sviluppa in quattro capitoli, che non presentano una semplice scansione cronologica.

Nel primo capitolo Francesca Fauri si sofferma sulle dinamiche che portarono alla formazione di un mercato internazionale del lavoro. Questo, infatti, «[...] stava diventando globale grazie a due caratteristiche imprescindibili di quest'epoca: 1) la libera circolazione della manodopera; 2) i progressi tecnologici nel campo dei trasporti»7.

La prima caratteristica, afferma l'autrice, era dovuta a contingenze quanto mai favorevoli: «[...] furono certamente anni irripetibili quanto alla possibilità di ricercare liberamente una meta migliore da raggiungere e che tenesse fede alle speranze di chi partiva ${ }^{8}$. Al successo di questa circolazione contribuì certamente un secondo fattore: «Questi furono gli anni in cui si compì la rivoluzione dei trasporti: ferrovie e navi a vapore sempre più veloci resero il mondo sempre più piccolo e permisero ai lavoratori di viaggiare separatamente e a prezzi in continuo ribasso»9.

L'autrice offre poi un compendio delle concause che animarono le migrazioni ottocentesche; rifiutando qualsiasi causa univoca, Fauri preferisce individuare quattro ragioni dei movimenti migratori applicabili a tutti i paesi coinvolti: il differenziale salariale (fra paese di partenza e paese di destinazione); la spinta demografica dovuta all'aumento della popolazione; la catena migratoria, in ragione della quale le migrazioni precedenti influenzarono quelle successive; la politica migratoria stabilita dai paesi di destinazione e di partenza.

Chiude il capitolo una ricognizione delle diverse valutazioni - operate dalle commissioni d'immigrazione così come dalla letteratura scientifica - dell'impatto economico dell'immigrazione negli Stati Uniti.

Il secondo capitolo è dedicato all'analisi dell'iter burocratico che dovevano intraprendere gli emigranti italiani per poter partire. L'autrice si sofferma anche sulla condizione dei trasporti marittimi in Italia e su una disamina dei flussi migratori in

\footnotetext{
5 LEVI, Primo, Il sistema periodico, Torino, Einaudi, 1975.

${ }^{6}$ CASSOLA, Carlo, Il taglio del bosco, Torino, Einaudi, 1959.

7 FAURI, Francesca, Storia economica delle migrazioni italiane, Bologna, Il Mulino, 2015, p. 15.

8 Ibidem.

9 Ibidem.
} 
uscita (riportati per regione e per provincia) - contestualizzata nella letteratura esistente - offrendo una grande quantità di dati.

Un ampio spazio è dedicato al ruolo delle rimesse. Francesca Fauri mette in evidenza la capacità di risparmio degli emigranti italiani, testimoniata dall'impressionante mole delle rimesse italiane. Ciò era dovuto allo spirito di sacrificio dell'emigrante italiano, che ne faceva: «[...] l'agente di una missione lucrativa a tempo determinato concordata con la famiglia di appartenenza» ${ }^{10}$. Gli effetti macroeconomici furono quelli di «[...] contribuire al successo della politica monetaria italiana che riuscì a realizzare un consistente apprezzamento della lira e la conversione del debito pubblico» ${ }^{11}$. Sotto il profilo microeconomico, invece, le rimesse vennero impiegate «[...] per comprare case (o ristrutturarle) e terre, ma anche per saldare i debiti della famiglia e aumentare la spesa per migliorare sensibilmente il livello di consumi primari, alimentari e vestiario» ${ }^{2}$. L'autrice afferma che: «[...] si trattò dunque di investimenti conservativi, che tendevano a difendere l'ordine esistente nella famiglia rurale e il suo ambiente culturale» ${ }^{13}$.

Il terzo capitolo ha un’impostazione quantitativa: è dedicato alla definizione delle rotte migratorie italiane. Fauri si sofferma in particolare sul caso degli Stati Uniti, dell'Argentina, del Brasile e dell'Africa mediterranea, numericamente ed economicamente significativi.

Se i primi capitoli sono dedicati al periodo precedente il primo conflitto mondiale, il quarto tratta del periodo fascista e del secondo dopoguerra. Durante il ventennio «[...] l'emigrazione venne [...] sempre meno tollerata, era considerata una sottrazione di elementi validi all'economia nazionale, all'esercito, alla vitalità biologica del paese (la fertilità era vista come arma) e pertanto partire venne reso sempre più costoso e difficile»14. L'obiettivo del regime era quello di reindirizzare i flussi verso la colonizzazione interna e i possedimenti africani. «Dal punto di vista economico e commerciale» sottolinea l'autrice «l'impero coloniale fu poco redditizio e molto costoso (sia per la guerra di conquista sia nella gestione amministrativa)»15.

Del resto il fenomeno migratorio, quando veniva impedito da misure politiche, trovava un suo sfogo nell'emigrazione clandestina, dal momento che

\footnotetext{
10 Ibidem, p. 89.

${ }^{11}$ Ibidem, p. 96.

12 Ibidem, p. 98.

13 Ibidem, p. 99.

14 Ibidem, pp. 172-173.

${ }^{15}$ Ibidem, p. 177.
} 
[...] i fattori che inducevano all'esodo illegale e alla sua tolleranza erano numerosi e ben più complessi della semplice relazione quantitativa tra la domanda e l'offerta di braccia, e rimandavano soprattutto alla politica migratoria ufficiale, al suo meccanismo burocratico e alle condizioni di accoglienza riservate agli stranieri ${ }^{16}$.

La seconda parte del capitolo è dedicata all'emigrazione italiana nel dopoguerra. Viene qui ricostruita la storia e l'attività del Comitato provvisorio intergovernativo per il movimento dei migranti dall'Europa (CPIMME), successivamente divenuto permanente (CIME). L'autrice prende poi in esame gli accordi bilaterali per regolare l'emigrazione lavorativa, con particolare attenzione alle mete europee, soffermandosi anche sugli ultimi anni.

Fauri evidenzia nel volume come sia sorta - in ragione della forte propensione migratoria italiana - una vera e propria «cultura della migrazione» ${ }^{17}$; sottolinea come, nella maggior parte dei casi, alla base della scelta di partire vi fossero un insieme di concause e quasi mai una decisione individuale: quella di partire era una scelta condivisa «[...] con obiettivi di risparmio definiti» ${ }^{18}$.

La storia migratoria italiana si sottrae ai modelli generali dimostrando di essere estremamente sfaccettata al suo interno; i modelli interpretativi proposti da Fauri respingono «[...] spiegazioni fondate su un'unica causa, evitando rigide delimitazioni teoriche»19. L'opera rappresenta un quadro generale dell'emigrazione sotto l'aspetto economico. Il maggior merito dell'autrice è quello di avere raccolto in queste pagine un grande numero di dati - molto utili si rivelano in questo caso le tabelle e i grafici di cui è costellato il libro - accompagnandoli con puntuali riferimenti storiografici.

Le statistiche presentate in questo libro rappresentano il quid in più di questo saggio: come sottolineato dall'autrice, per quanto possano essere oggetto di critica (sono basati sui documenti rilasciati dai prefetti e non sulle effettive partenze), rappresentano un'approssimazione realistica e sono i dati più completi attualmente a disposizione dei ricercatori.

${ }^{16}$ RINAURO, Sandro, op. cit., p. XIII.

${ }_{17}$ FAURI, Francesca, Storia economica delle migrazioni italiane, cit., p. 221

${ }^{18}$ Ibidem, p. 222.

19 Ibidem, p. 226. 


\section{* L'autore}

Jacopo Bassi ha conseguito la laurea specialistica in Storia d'Europa presso l'Università degli Studi di Bologna. Nel corso dei suoi studi si è occupato di storia della Grecia e dell'Albania in età contemporanea e di storia e istituzioni della Chiesa ortodossa. Ha lavorato per le case editrici Éditions des femmes, Il Mulino e Zanichelli.

URL: < http://www.studistorici.com/2009/o2/24/jacopo_bassi/ >

\section{Per citare questo articolo:}

BASSI, Jacopo, «Recensione: Francesca FAURI, Storia economica delle migrazioni italiane, Bologna, II Mulino, 2015, 233 pp.», Diacronie. Studi di Storia Contemporanea : Un bilancio della scommessa democratica della Chiesa cattolica, 29/06/2016,

URL:< http://www.studistorici.com/2015/06/29/bassi_numero_26/ >

\section{Diacronie Studi di Storia Contemporanea 3 www.diacronie.it}

Risorsa digitale indipendente a carattere storiografico. Uscita trimestrale. redazione.diacronie@hotmail.it

Comitato di redazione: Jacopo Bassi - Luca Bufarale - Elisa Grandi - Antonio César Moreno Cantano - Deborah Paci - Fausto Pietrancosta - Alessandro Salvador - Matteo Tomasoni - Luca Zuccolo

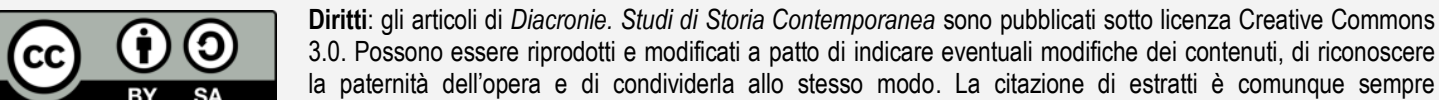
la paternità dell'opera e di condivider
autorizzata, nei limiti previsti dalla legge. 